\title{
Validación de un Inventario de Comportamientos Agresivos en el Tránsito
}

\section{Validation of an Aggressive Driving Behaviors in Traffic Inventory}

\author{
Gabriel Dorantes Argandar
}

Universidad Autónoma del Estado de Morelos

\section{Nota sobre el Autor}

Gabriel Dorantes Argandar. Centro de Investigación Transdisciplinar en Psicología de la Universidad Autónoma del Estado de Morelos.

Esta investigación fue financiada con recursos del autor.

Remita cualquier duda sobre este artículo al siguiente domicilio: Pico de Orizaba No. 1 Col. Los Volcanes. Cuernavaca, Morelos, México 62350. Correo electrónico: 


\section{Resumen}

Anualmente fallecen más de 20 mil personas en accidentes viales. (Cervantes-Trejo, 2009). Asimismo, otras 700 mil sufren daños que requieren de hospitalización, así como 40 mil personas sufren lesiones que terminan en algún tipo de discapacidad (Cervantes-Trejo, 2009). Sin embargo, estos números rápidamente van en aumento año tras año (Cervantes-Trejo, 2009). Una de las causas principales de la accidentalidad es la agresividad vial. Para este estudio, la agresividad vial se define en términos de poca consideración, conducción de riesgo y manifestación de frustración/enfado. Para ello, este estudio estableció como objetivo principal el construir el Inventario de Comportamientos Agresivos en el Tránsito que midiera el constructo. Se planteó la siguiente pregunta de investigación: ¿Es válido y fiable el inventario generado? Esta investigación fue una validación local en Cuernavaca, Morelos, con 2454 participantes. Se hicieron tres estudios. El primero encontró que hubo más ítems generados en manifestación de frustración/enfado que para poca consideración y conducción de riesgo. El segundo estudio encontró una alta validez de constructo y aceptable consistencia interna. El tercer estudio encontró una alta validez de constructo y buena consistencia interna. Sin embargo, no se evaluaron la validez de contenido, convergente ni divergente. Se concluye que el Inventario de Comportamientos Agresivos es un instrumento válido pero requiere de estudios adicionales para evaluar sus propiedades psicométricas en una muestra representativa.

Palabras clave: agresividad vial, Inventario de Comportamientos Agresivos en el Tránsito, poca consideración, conducción de riesgo, manifestación de frustración/enfado 


\section{Abstract}

Over 20000 people die due to car accidents (Cervantes-Trejo, 2009). Moreover, other 700000 suffer injuries that result in hospitalization, as well as 40000 individuals end up with some sort of disability (Cervantes-Trejo, 2009). Alarmingly, Cervantes-Trejo (2009) reported that this problematic is rising every year. Aggressive driving is one of the main causes of traffic accidents. In this study, aggressive driving is defined in terms of inconsiderateness towards others, risky driving and frustration/hostility behaviors. The aim of this study was to create and validate an instrument to assess the construct. We intended to answer the following research question: Is the Aggressive Driving Behavior Inventory valid and reliable? This is a local validation study in Cuernavaca, Morelos in Mexico. We carried out three studies sampling 2454 participants. In the first study, we found more items in the dimension frustration/hostility behaviors rather than the ones in the other dimensions. In the second one, we discovered a high construct validity and acceptable internal consistency of the instrument. In the third one, we found that the measure obtained a high construct validity and good internal consistency. Nonetheless, we didn't assess the content, convergent and divergent validity. We concluded that the Aggressive Driving Behavior Inventory is valid measure but needs further assessment of its psychometric soundness in a representative sample.

Keywords: aggressive driving, Aggressive Driving Behavior Inventory, inconsiderateness towards others, risky driving, frustration/hostility behaviors 


\section{Introducción}

La mayor parte de la problemática social (inseguridad, violencia, victimización resentimiento social, ira, individualismo) encuentra su fuente en el estrés al que el ser humano se somete cotidianamente. Selye (1978) establece que conducir un vehículo automotor es capaz de estresar al ser humano debido a su variada combinación de factores, tales como la urbanización y el aglomeramiento de seres humanos, pero él mismo admite que la intricada complejidad de factores causantes del estrés requieren de una labor muy delicada y minuciosa para poder identificarlos. Tal como establecen Hinojosa-Reyes, Jiménez-Sánchez, Hernández-Hernández y Campos Alanís (2012), México está entre las naciones de mayor índice de mortalidad a nivel mundial. Esto es debido a los accidentes de tránsito y, particularmente, en América Latina se encuentra en el segundo lugar, antes de Guyana, quien es el primero. La primer causa de muerte entre adolescentes son los accidentes viales, y sobre dos millones de jóvenes entre 12 y 18 años sufren lesiones por eventos de esta naturaleza; anualmente fallecen más de 20 mil personas y otras 700 mil sufren daños que requieren de hospitalización, así como 40 mil personas sufren lesiones que terminan en algún tipo de discapacidad (Cervantes-Trejo, 2009). El Estado de Morelos sufre una tasa de mortalidad de 12.3 por cada 100 mil habitantes debido a accidentes en la movilidad, y de estos el 93.7\% se llevan a cabo en zonas urbanas y suburbanas (Observatorio Nacional de Lesiones, 2013). La accidentalidad no es un fenómeno individual a nivel del conductor, es una problemática social y un fenómeno que debe de ser observado como un sistema de interacciones. Un accidente en la movilidad se define como un evento en el que uno o más individuos sufren un daño, ya sea material o humano, y existe un riesgo para la integridad física de por lo menos una 
persona, dentro del contexto de conducir un vehículo automotor. Cuevas Colunga, Villegas Villegas, Mayoral Grajeda, y Mendoza Díaz (2014) describen un accidente de tránsito como un percance vial que se presenta repentinamente y sin ser esperado, el cual se determina por: (1) las condiciones del contexto y (2) los actos irresponsables de los conductores que son parcialmente previsibles; y se atribuyen a factores humanos, otros vehículos automotores, condiciones del clima y el medio ambiente, mala señalización e infraestructura, los cuales ocasionan lesiones de distintos niveles de gravedad y/o pérdida de vida, así como secuelas que pueden ocasionar una discapacidad, perjuicios materiales y daños a terceros. Al mirar la estadística antes presentada, se puede asumir que el contexto en el cual se encuentran inmersos los habitantes de la ciudad de Cuernavaca es aversivo para su bienestar. Diversos estudios realizados previamente en dicha ciudad demuestran que el entorno es estresante (Dorantes-Argandar, Tortosa-Gil, \& Ferrero-Berlanga, 2016; LimaAranzaes, Juárez-García, \& Arias-Galicia, 2012; Treviño-Siller, Híjar, \& Mora, 2011). Además, hay una compleja relación entre el estrés y la agresividad (Dorantes-Argandar, Cerda-Macedo, Tortosa-Gil, \& Ferrero Berlanga, 2015a). Asimismo, también existe una asociación del estrés y la agresividad con el comportamiento prosocial (Dorantes-Argandar, Cerda-Macedo, Tortosa-Gil, \& Ferrero Berlanga, 2015b).

El comportamiento agresivo en la movilidad es una problemática real en México. El entorno mexicano se ha vuelto más violento para el ciudadano común, y por ende el ciudadano común se ha vuelto agresivo con su entorno. Esta es una realidad que se repite a nivel local, en la ciudad de Cuernavaca, Morelos. Esta situación precisa la generación de une herramienta que permita evaluar el comportamiento agresivo en el tránsito de una manera consistencia y válida. La revisión bibliográfica aquí presentada nos permite determinar que el comportamiento agresivo en la movilidad posee dos componentes principales: poca consideración y/o mucha frustración e 
irritación hacia los demás, y un estilo de conducir que pone en peligro a todos los individuos inmersos en el entorno. El propósito del presente estudio es diseñar un inventario que sea capaz de evaluar la agresividad vial en la ciudad de Cuernavaca, Morelos. Además de esto, dado que no hay una gran cantidad de estudios sobre agresividad vial en México, es necesario determinar si la concepción teórica de agresividad se ajusta a la manifestación de la agresividad en México. Se ha encontrado que el comportamiento agresivo está intrínsecamente relacionado con los entornos de movilidad que ocasionan estrés (Houston, Harris, y Norman, 2003). Por tanto, se pretende entender la dinámica social que se observa en la movilidad cuernavacense en relación a la agresión vial. Es de esperar que los conductores de esta ciudad hayan desarrollado un alto nivel de agresividad vial a manera de estrategia de afrontamiento (Lazarus \& Folkman, 1984) con el fin de hacer frente a las vicisitudes del contexto. Este estudio tiene como objetivo el diseñar una escala que sea capaz de evaluar la agresividad vial en Cuernavaca. Por tanto, la pregunta de investigación de nuestro estudio se basa en si ¿Es válido y confiable el Inventario de Comportamientos Agresivos en el Tránsito?

\section{Revisión de la Literatura}

\section{La Agresividad}

La agresividad se define como agresión a todo aquel comportamiento indeseado que es percibido por la persona que es blanco de dicho comportamiento como intrusivo y generador de daño (Awopetu Ronke \& Igbo Happiness, 2015). Hennessy y Wiesenthal (2002) definen la agresión como "cualquier comportamiento que tengan la intensión de dañar a otros, ya sea físicamente, psicológicamente o emocionalmente" (p. 411). 
La agresividad como rasgo y como estado. La agresividad como rasgo se define como la tendencia general a desencadenar comportamientos agresivos a manera constante de expresión en diferentes contextos de la vida de un individuo dado. Se entiende que es de rasgo por estar presente a través de los rubros vitales del individuo (Locke, 2009; Przepiorka, Blachnio, \& Wiesenthal, 2014), debido al vínculo que tiene con otras variables de personalidad como la autoestima (Strandell, 2016), y la impulsividad (Dorantes-Argandar \& Ferrero-Berlanga, 2017), y por ser reconocida como una de las fuerzas principales que gobiernan el comportamiento humano (Seligman, 2002). La agresión como estado se define como el acto mismo y unitario, la evocación de un solo comportamiento que tiene como fin causar daño.

La agresividad como rasgo es un fenómeno ampliamente estudiado en diversos ámbitos. En la Tabla 1 se resumen algunos de los campos en los que se aborda el tema de la agresión.

\section{La Movilidad}

El comportamiento en la movilidad es un área de estudio que poco ha impactado a los países de tradición latinoamericana. Éste es un tema de interés que poco a poco ha ido adquiriendo fuerza en la literatura científica (Dorantes-Argandar et al., 2015a; Rodríguez, Virguez, Rodríguez, \& Behrentz, 2016). Sin embargo, se puede aprovechar mucho de los avances obtenidos en otros países para entender esta dinámica y buscar explicarla a nivel local.

Los comportamientos básicos en la movilidad. Abdu, Shinar, y Meiran (2012) puntualizaron que existen dos tipos básicos de comportamientos en la movilidad: (1) aquel comportamiento basado en la habilidad misma del conductor, y (2) aquel comportamiento basado en el cálculo de riesgo que realiza el conductor en una situación determinada. El argumento básico de estos autores es que el primero no depende del estado emocional del individuo, sólo de su 
capacidad de operar el vehículo en una situación determinada; el otro depende de un cálculo cognitivo. Esto hace que el comportamiento en la movilidad dependa de todas las estructuras que están adheridas al proceso de evaluación (Lazarus \& Folkman, 1984).

Los accidentes en la movilidad. El desencadenamiento de un accidente de tránsito se puede deber a un rango de factores, que va desde la experiencia misma del conductor, el historial de ofensas y errores cometidos por el mismo, así como faltas a la ley que tienen un componente agresivo subyacente (Al Reesi et al., 2013). Dorantes-Argandar et al. (2016) definen los tres factores que hacen mella en el bienestar de los individuos en el entorno de la movilidad: (1) la autoridad, el respeto, la prudencia y el orden social, (2) el respeto por las normas, la infraestructura y el medio ambiente, y (3) elementos dentro del vehículo y la visibilidad. Existen estudios que demuestran que el clima y la geometría misma de la cinta asfáltica tienen influencia en el comportamiento del conductor (Hamdar, Qin, \& Talebpour, 2016), así como distractores tales como el uso del teléfono móvil (Oviedo-Trespalacios, Haque, King, \& Washington, 2016). También es importante considerar que factores climáticos ajenos al ser humano también tienen influencia en el comportamiento del conductor y el riesgo de tener un accidente, como lo son la lluvia, la neblina, los deslaves, o en su caso, los desastres naturales. Esto permite contextualizar el riesgo y la accidentalidad misma desde una perspectiva objetiva y no sólo desde el punto de vista psicológico.

Aquellos individuos que están más inclinados a no respetar las normas tienen mayores niveles de aceptación a beneficiarse a costa de otros, pero también tienen los menores niveles de enfado y el mayor nivel de apoyo a la realización de comportamientos agresivos (Bailey, Lennon, 
\& Watson, 2016). Es posible que la alta proliferación de comportamiento agresivo equivalga a un incremento en el comportamiento antisocial.

Las conductas de riesgo en la movilidad. El comportamiento de riesgo en el tránsito está relacionado con el enfado, la búsqueda de sensaciones, la sensación de urgencia y un bajo nivel de premeditación y perseverancia en las actividades diarias (Bachoo, Bhagwanjee, \& Govender, 2013). Las medidas de comportamiento de riesgo en la movilidad pueden llegar a incluir hasta evaluación subjetiva de la velocidad, profundidad del pedal de aceleración, y velocidad de rotación del volante (Ba, Zhang, Salvendy, Cheng, \& Ventsislavova, 2016). El conductor en general suele sobreestimar su propia capacidad para operar un vehículo automotor (Amado, Arıkan, Kaça, Koyuncu, \& Turkan, 2014), por lo que el estudio de los factores psicológicos involucrados en la movilidad son de suma importancia. La baja perseverancia en las actividades diarias puede estar relacionado con bajos niveles de bienestar tanto objetivo como subjetivo, así como factores psicosociales y de personalidad como la autoeficacia, la esperanza, la autoestima, etc. El comportamiento de riesgo en la movilidad está relacionado con la inhabilidad de manejar emociones, mal manejo del estrés y poca capacidad de manejo interpersonal (Arnau-Sabatés, SalaRoca, \& Jariot-Garcia, 2012). Algunas instancias de enfado tras el volante son respuestas emocionales a cogniciones de naturaleza moral que los conductores hacen sobre la atribución de los comportamientos de otros (Bailey et al., 2016). De ellas, la hostilidad se construye a través de rasgos de la personalidad como el antagonismo y el afecto negativo (Beanland et al., 2014). Beck y Watters (2016) consideran al uso del celular mientras se conduce como un comportamiento ajeno a el comportamiento agresivo y/o el comportamiento de riesgo, pero encuentran que existe fuerte asociación entre estos tres elementos. 
Los conductores que manifiestan llevar prisa más a menudo cuando conducen suelen desencadenar comportamientos que se consideran de riesgo así como de agresión (Beck, Daughters, \& Ali, 2013). Es posible que dicha manifestación esté asociada a la impaciencia como rasgo de personalidad. La intolerancia a las situaciones estresantes está caracterizada por un poco o nula habilidad para evitar ciertas respuestas comportamentales de naturaleza desadaptativa de cara a circunstancias amenazantes o de reto, y que son absorbidas por la atención y las emociones negativas (Beck et al., 2013) asociadas al proceso de evaluación (Lazarus \& Folkman, 1984). ¿Dónde termina el comportamiento agresivo y se convierte en comportamiento de riesgo? Al llevar a cabo comportamientos de naturaleza riesgosa, ¿no se está agrediendo al tejido social? Una buena clasificación de comportamiento antisocial podría incluir el comportamiento agresivo y el de riesgo. El comportamiento agresivo difiere del comportamiento de riesgo debido a la intención de hacer daño, más que sólo poner en peligro la vida de otros (Al Reesi et al., 2013; Deffenbacher, Lynch, Filetti, Dahlen, \& Oetting, 2003; Deffenbacher, Deffenbacher, Lynch, \& Richards, 2003; Richer \& Bergeron, 2012), aunque es válido postular que el comportamiento agresivo es en sí un comportamiento de riesgo.

El comportamiento agresivo en la movilidad. Al parecer la necesidad de reaccionar y llevar a cabo una secuencia de toma de decisiones en tiempo real, debido a la repentina aparición de un obstáculo o a una situación determinada que exija detener la locomoción del vehículo puede ser causa de frustración, y, por lo tanto, evocar un comportamiento que sea de naturaleza agresiva. El comportamiento agresivo no sólo está vinculado con un rasgo de personalidad, sino que también está relacionado con causas situacionales que pueden ser muy particulares.

La ira al volante es definida como "el uso de un vehículo automotor para manifestar comportamiento agresivo, lo cual contribuye a la violencia vial, y por lo general es desencadenada 
sin previa meditación" (Brewer, 2000, p.49). Dos de los factores que se ha encontrado que estén vinculados con la agresión al volante son la falta de confianza social y el desagrado generalizado, lo cual lleva a un comportamiento de transgresión de las normas sociales y legales, e incluso a comportamiento agresivo hacia otros (Houston et al., 2003). Las asociaciones de cognición que son de naturaleza hostil pueden ser primadas y por lo tanto inmediatamente influyen en las respuestas automáticas, pues es posible que los procesos de aprendizaje asociativo de alguna manera hayan sido diferentes para individuos que tienden a comportarse agresivamente (Blankenship \& Nesbit, 2013). Björklund (2008) encuentra que el impedimento a la locomoción del vehículo, como la aparición repentina de un obstáculo, desencadena sentimientos de frustración en los individuos, lo que generalmente deviene en un comportamiento agresivo como primera opción de respuesta en un proceso de toma de decisiones.

Shinar (2007) define el comportamiento agresivo en la movilidad de la siguiente manera: un síndrome de comportamientos instrumentales motivados por la frustración, los cuales son manifestados en (1) o nula consideración e irritación hacia otros conductores (reducir la distancia entre vehículos, utilizar inadecuadamente la intensidad de las luces, y utilizar la bocina o claxon), y (2) conducir deliberadamente de una manera que pone en peligro al conductor y a aquellos que lo rodean para ahorrar tiempo a costa del bienestar de otros (no respetar los semáforos en rojo o señales de detener la marcha, obstruir el paso de otros, maniobrar deliberadamente hacia los lados a manera de agresión). Uno es independiente del otro y no suelen correlacionar estadísticamente (Arnau-Sabatés et al., 2012).

El comportamiento agresivo en la movilidad y los rasgos de personalidad. Los conductores con un alto nivel de enfado de rasgo tienen más probabilidad de atribuir situaciones hostiles de tránsito y por lo tanto conducir más rápido, cometer más errores y ponerse a sí mismo y a los 
demás en más riesgo en general (Abdu et al., 2012). El proceso de atribución y el sesgo de atribución hostil está ampliamente discutido en referencia a diferentes contextos (Bonus, Peebles, \& Riddle, 2015; Britt \& Garrity, 2006; Castellani et al., 2014; Helfritz-Sinville \& Stanford, 2014; Martins, 2013; Werner, 2012; Wu, Zhang, Chiu, Kwan, \& He, 2014). Las cogniciones hostiles que pueden o no ser de naturaleza explícita pueden ser el mecanismo sobre el cual se construye la dinámica entre el enfado como rasgo de personalidad y el comportamiento agresivo (Blankenship \& Nesbit, 2013).

Todo lo antes expuesto sugiere una fuerte implicación de variables que son de personalidad, como la autoestima y el apego a las normas, durante la ejecución de una serie de maniobras en un sistema de toma de decisiones que se desencadena en tiempo real, lo cual significa que las personas que realizan estos comportamientos con intención son diferentes de aquellos que sólo carecen de pericia en su estilo de conducir (Hennessy \& Wiesenthal, 2002). El comportamiento agresivo al conducir está relacionado con el estilo de auto-presentación, de tal manera que aquellos individuos que desean ser percibidos como intimidantes, desagradecidos, y modelos rudos y dominantes a seguir, son más agresivos en su estilo de conducir (Bassett, Cate, \& Dabbs, 2002). Sin embargo, también se encuentra que los conductores que manifiestan tener más prisa tienen mayores niveles de frustración e impaciencia que otros conductores, así como enfado e intolerancia al estrés (Beck et al., 2013). Esto hace que podamos hablar de dos estilos de conducir agresivos: el impaciente y el dominante.

La definición de comportamiento agresivo debe de excluir todos los actos accidentales que llevan a ocasionar un daño, como perder el control de un vehículo y que esto tenga como resultado un daño, pero incluye todo comportamiento que tenga la intención de hacer daño aunque no logre su objetivo (Awopetu Ronke \& Igbo Happiness, 2015). Sin embargo, es preciso entender que 
operar un vehículo automotor cuando no se está propiamente capacitado para ello, o no se está en condiciones óptimas para hacerlo, es un elemento que debe de ser considerado dentro de la argumentación de la definición. Si un individuo dado tiene la plena conciencia de que conduce su vehículo bajo los efectos del alcohol, ¿Está agrediendo al tejido social? ¿Y si no tiene la consciencia de que no está debidamente capacitado para operar el vehículo? La agresividad en el tránsito asociado con los rasgos de personalidad, se considera manifiesta a través de todo aquel comportamiento que exprese la poca o nula consideración que el conductor tiene hacia el entorno y los individuos inmersos en él, que manifieste un alto nivel de frustración a los obstáculos que la dinámica de la movilidad coloca frente al conductor, y un marcado estilo de conducir que incrementa el riesgo al que el conductor mismo y aquellos individuos que lo rodean están de padecer un accidente. Tres son los rasgos de personalidad que más claramente se asocian con el comportamiento agresivo en la movilidad (Beanland et al., 2014): el Antagonismo, la Desinhibición y el Afecto Negativo.

Aspectos del comportamiento agresivo. El comportamiento agresivo debe de estar compuesto por aspectos que permitan generar un constructo a través del cual se pueda entender su complejidad. Los conductores agresivos suelen estar predispuestos a comportarse de una manera que es menos prevalente en conductores que no lo son, y no es una situación en la que dichos individuos carezcan de los esquemas comportamentales requeridos (Beck, Wang, \& Mitchell, 2006). El conductor agresivo sabe que su comportamiento es agresivo y suele poner su beneficio por encima del de otros: tiene los esquemas comportamentales requeridos, pero elije no usarlos. Estas concepciones de la agresividad en la movilidad y el conductor agresivo suenan más a comportamiento antisocial, más que meramente agresivo. La definición más aceptada de comportamiento agresivo en el tránsito (Shinar, 2007) incluye el elemento de acción voluntaria 
del individuo que busca beneficiarse a costa de otros. Gidron et al. (2014) consideran que el comportamiento agresivo se divide en "agresión hacia afuera", la cual incluye la manifestación comportamental del enfado, y en "agresión hacia adentro", la tendencia a inhibir tales expresiones. Özkan, Lajunen, Chliaoutakis, Parker, \& Summala (2006) encuentran que son tres los componentes del comportamiento agresivo: violaciones agresivas, violaciones ordinarias, y errores al conducir. La revisión bibliográfica realizada para este estudio encuentra que hay diversas posturas teóricas para caracterizar el comportamiento agresivo al volante, y la evidencia empírica demuestra que dicho constructo es diferente incluso cuando se aplica un mismo instrumento transculturalmente ( $\mathrm{Li}$, Yao, Jiang, \& Li, 2014). Por ejemplo, las diferentes validaciones del instrumento de Deffenbacher et al. (2002) arroja diferentes estructuras factoriales en los diferentes países en los que ha sido validado.

Para propósitos de éste trabajo se realizó una búsqueda exhaustiva de otros instrumentos que hubieran sido diseñados para medir la conducción agresiva. De hecho, ya se sabía que no había una escala que hubiera sido diseñada ex profeso para el contexto mexicano, mucho menos para el contexto local. En general se considera que el comportamiento agresivo se compone por agresión verbal, expresión personal de agresión física, uso del vehículo para expresar enfado, agresión desplazada, y expresión adaptativa/constructiva (Deffenbacher et al., 2002); sólo conducción agresiva (Harris et al., 2014; Houston, et al., 2003); expresión agresiva física y verbal, expresión adaptativa/constructiva, y uso del vehículo para la expresión de la agresión (Sârbescu, 2012);expresión adaptativa/constructiva, expresión personal y física de la agresión, expresión verbal de la agresión, y uso del vehículo para expresar la agresión (Ge, Qu, Zhang, Zhao, \& Zhang, 2015; Gras et al., 2015; Stephens \& Sullman, 2014); sólo comportamiento agresivo/hostil (Clapp et al., 2011); agresión y exceso de velocidad (Bener, Özkan, \& Lajunen, 2008) ; control, enfado 
hacia adentro, agresión física-objetos, expresión directa, discusión acalorada, agresión verbal, comunicación recíproca y tiempo fuera (Deffenbacher et al., 1996); expresión verbal de la agresión, expresión personal y física de la agresión, uso del vehículo para expresar agresión, agresión desplazada, y expresión adaptativa/constructiva de la agresión (Herrero-Fernández, 2011b); y conducción agresiva (Willemsen, Dula, Declercq, \& Verhaeghe, 2008). Aunque la definición de (Shinar, 2007) parece ser la más aceptada mundialmente, no parece haber un consenso sobre la estructura factorial del comportamiento agresivo al volante.

Escalas que miden el comportamiento agresivo. Aparentemente los instrumentos más utilizados en el mundo para evaluar el comportamiento son: (1) el Driving Behavior Questionnaire (DBQ), pero éste instrumento sólo tiene un componente de conducción agresiva, pues evalúa todo el espectro del comportamiento en la movilidad; (2) el Driving Anger Scale; (3) un instrumento importante en la medición de la expresión de la agresividad en la movilidad es el Driving Anger Expression Inventory; (4) y también existe el Driving Behavior Survey, que también es de uso frecuente pero éste último, al igual que el DBQ, sólo aborda el comportamiento agresivo como un componente de un espectro más amplio. En la Tabla 2 se presentan las publicaciones en las que aparecen estos instrumentos.

El inventario de comportamientos agresivos de Dorantes Argandar. En literatura publicada en países latinoamericanos, se encontró que Poó (2014) hizo una validación de escalas de agresividad vial en Argentina. Sin embargo, hasta la fecha no se han realizado validaciones de escalas de agresividad en México. Por tanto, se considera importante realizar una escala que se ajuste a las particularidades de la movilidad que existen hoy en día y que constituyen una problemática muy diferente a la que existe en otros países. Este inventario aborda a los 
comportamientos agresivos como una construcción multidimensional que busca agrupar todas las manifestaciones de la agresión que un conductor puede llevar acabo al operar un vehículo automotor, en términos de poca consideración para con otros, manifestaciones de frustración/enfado, y conducción deliberadamente equivocada. Este inventario considera los comportamientos agresivos como un constructo multidimensional tomando en cuenta los aspectos considerados por Shinar (2007). Por consiguiente, en este inventario se define la agresividad vial en términos de poca consideración, manifestaciones de frustración e irritación, y conducción deliberadamente equivocada.

Aspectos del inventario de comportamientos agresivos de Dorantes Argandar. El Inventario de Comportamientos Agresivos en el Tránsito (ICAT) busca abordar 3 aspectos de comportamientos principales: (1) poca consideración, (2) manifestaciones de frustración/enfado, y (3) conducción de riesgo. La poca consideración para con otros se define como la expresión de mala educación vial o poca consideración para con otros conductores y/o peatones (Bailey et al., 2016; Deffenbacher, 2011; Taubman - Ben-Ari \& Skvirsky, 2016; Vanlaar, Simpson, Mayhew, \& Robertson, 2008). Se entiende por manifestaciones de frustración/enfado todos aquellos comportamientos que buscan expresar este sentir en el conductor (Bailey et al., 2016; Deffenbacher, 2016; Deffenbacher, Deffenbacher, et al., 2003; Deffenbacher et al., 2002, 1996; Gidron et al., 2014; Kovácsová, Lajunen, \& Rošková, 2016; Kovácsováet al. 2014; Lajunen \& Parker, 2001; Smith et al., 2006; Walitzer et al., 2015). La conducción de riesgo es todo aquel comportamiento que se lleva a cabo, no sólo sabiendo que está expresamente prohibido, sino que también incrementa la probabilidad de causar daño a uno mismo y/o a otros (Al Reesi et al., 2013; Bachoo et al., 2013; Beck, Ali, \& Daughters, 2014; Constantinou, Panayiotou, Konstantinou, Loutsiou-Ladd, \& Kapardis, 2011; Deffenbacher, Deffenbacher, et al., 2003; Dula \& Geller, 2003; 
Hsieh et al., 2015; Pearson, Murphy, \& Doane, 2013; Richer \& Bergeron, 2012; Rosenbloom \& Wultz, 2011).

\section{Objetivos y Alcances}

El presente estudio tiene como objetivo presentar un instrumento que sea capaz de evaluar el comportamiento agresivo tal y como se vive en la ciudad de Cuernavaca, Morelos. La presente investigación es, por lo tanto, un estudio de validación. Busca generar la validez de constructo para una escala que se construyó para la población de dicha ciudad. El uso de este instrumento en otras ciudades de México se recomienda, pero se sugiere realizar un estudio previo de validación local para asegurarse que los criterios presentados en esta investigación se cumplen.

\section{Método}

\section{Diseño}

Esta investigación es de naturaleza cuantitativa, no-experimental, de corte transversal, confirmatorio, ex post facto. Se realizó un levantamiento de datos en el área conurbada de la ciudad de Cuernavaca, la cual comprende los municipios de Cuernavaca, Temixco, Jiutepec y Emiliano Zapata, a través de un equipo de investigación construido específicamente para este propósito, a lo largo de un periodo 6 meses. La base de datos generada fue procesada a través de la paquetería estadística SPSS en su versión 19. Este esfuerzo estuvo constituido por tres estudios independientes a través de los cuales se pretende validar al Inventario de Comportamientos Agresivos en el Tránsito (ICAT).

\section{Participantes}


La validación de la escala se obtuvo a través de 3 muestras diferentes. La primera permitió evaluar la selección de ítems. Se encuestó a 247 individuos (52\% mujeres, $M=33.7, S D=14.1$ ) a través del equipo de investigación reportado en este estudio. Los participantes fueron elegidos a través de un muestreo no-probabilístico de conveniencia. Los encuestadores fueron seleccionados a través de un grupo de estudiantes de la licenciatura en Psicología, supervisados por el investigador principal de este proyecto. Dichos practicantes abordaban a sus vecinos, familiares y amigos que cumplieran con los criterios de inclusión establecidos para este estudio (ser mayor de edad y poseer licencia de conducir). Se ordenó que no se concentraran en su círculo social inmediato, y que se esforzaran por traspasar el perímetro de dicha esfera, a través de recomendaciones y contactos.

La segunda fase de la validación de constructo se realizó a través de una segunda muestra que estuvo compuesta por 444 individuos, de los cuales el $60.8 \%$ eran hombres y el $39.2 \%$ mujeres. La media de edad fue de 36.42 años con una desviación estándar de 9.80 años. Las ocupaciones fueron las siguientes: empleado (18\%), estudiante (11.9\%), comerciante (7.2\%), ama de casa (4.9\%) y otras (58\%). Los participantes fueron elegidos por un método no-probabilístico sistematizado, tal y como se describe en la primera fase.

La tercera fase de la validación de constructo, se realizó a través de una tercera muestra que estuvo compuesta por 1763 individuos, de los cuales el $62.9 \%$ eran hombres y el $37.1 \%$ mujeres. La media de edad fue de 34.15 años con una desviación estándar de 11.62 años. Las ocupaciones fueron las siguientes: estudiante (24\%), empleado (15.7\%), comerciante (5.5\%), ama de casa (3.8\%) y otras (51\%).Los participantes fueron elegidos por un método no-probabilístico sistematizado tal y como se describe en la primera fase. 
La validación de este instrumento se realizó en el área conurbada de la ciudad de Cuernavaca, Morelos, la cual comprende los municipios de Cuernavaca, Jiutepec, Temixco y Emiliano Zapata (Instituto Nacional de Estadistica y Geografía, 2010), debido a que la mancha urbana de la ciudad trasciende los límites municipales de Cuernavaca y la actividad social de los cuatro municipios es prácticamente el de una sola ciudad.

\section{Instrumento}

El propósito de este estudio es la generación de un instrumento cuantitativo que evalúe a la agresividad como rasgo a través de los comportamientos agresivos. Por esta razón, se presenta a continuación los tres estudios individuales que se llevaron a cabo para satisfacer algunos criterios de la validez y fiabilidad que puede tener un instrumento: validez de constructo y consistencia interna. Este instrumento busca evaluar la poca consideración (no ceder el paso, no respetar las filas), la expresión de la frustración/enfado (uso excesivo del claxon/bocina, realizar señas obscenas), y la conducción de riesgo (conducir a exceso de velocidad, circular en sentido contrario).

\section{Procedimiento}

El primer estudio fue diseñado para seleccionar los ítems, el segundo pretendía obtener la validez de constructo, y el tercero buscó confirmar dicha validez. Este procedimiento es el mismo encontrado en Dorantes-Argandar et al. (2016) y Dorantes-Argandar (2016) por lo que es posible que a través de él se encuentre una óptima validez de constructo. Fueron excluidos 29 participantes por usar el mismo tipo de respuesta en toda la escala. Asimismo, no fueron considerados en el análisis 26 participantes por contar con una distancia Malahanobis mayor al valor crítico de 46.8 . Por lo que se analizaron los datos en 381 participantes. 


\section{Análisis}

Se evaluó la fiabilidad de la solución final de componentes principales. Asimismo, Se seleccionaron los ítems en base a ciertos criterios y se analizó la validez de constructo. A continuación, se detallan cada una de ellos.

Selección de ítems. Para este estudio, se les entregó a los participantes un formato en papel que solicitaba 3 respuestas a la pregunta ¿Qué es lo que hace un conductor agresivo? Para posteriormente contabilizar las respuestas más frecuentes. Se agruparon los ítems en base a frecuencias, lo que indicaban si ese ítem debía ser considerado en el instrumento. Por ejemplo: el ítem 1 "señas obscenas" indicaba el aspecto de manifestación de frustración/enfado. El ítem 2 “impedir el paso" indicaba el aspecto de poca consideración. En esta fase sólo se seleccionaron los ítems que se someterían a análisis estadístico en la siguiente fase.

La validez de constructo. Hunter y Schmidt (citado en Parsian \& Dunning, 2009) puntualizaron que la validez de constructo, la cual es un concepto cuantitativo, se basa en las estimaciones estadísticas, fundamentalmente la relación entre la construcción del instrumento y el indicador de que se manifiesta de forma numérica. Además, Kane, DeVon y colegas (citado en Parsian \& Dunning, 2009) mencionaron que el procedimiento indica la cohesión entre un instrumento, el marco en el que se basa, y la exactitud con la que lo hace.

Análisis de componentes principales. Esto se realizó con SPSS 19paraexplorar la estructura de las dimensiones de la escala propuesta. Se revisaron los datos de los valores faltantes, los errores, la distribución de respuestas por participante, y los supuestos de normalidad univariados y multivariados. Se excluyeron a los participantes que usaron el mismo grado de 
respuesta en todos los ítems. Asimismo, se excluyeron a los participantes con una distancia Malahanobis mayor a 46.8 para cumplir con el criterio de normalidad multivariada.

Se realizó el análisis de componentes principales extrayendo la solución inicial de componentes en base a un valor Eigen mayor a uno. Del mismo modo, se analizó si los datos eran factorizables a través de la prueba de esfericidad de Bartlett y la medida de Kaiser-Meyer-Olkin de adecuación muestral(KMO). De acuerdo con Kaiser (1974) los datos son factorizables si la prueba de esfericidad de Bartlett es estadísticamente significativa y la KMO es superior al criterio mínimo. Asimismo, se verificaron que los ítems tuvieran una comunalidad superior a 0,3. De lo contrario fueron eliminados. Posteriormente, se obtuvo la solución final de componentes para explicar más del 40\% de la varianza. Asimismo, se usó una rotación ortogonal como la de Varimax.

\section{Fiabilidad.}

Consistencia interna. Con el fin de evaluar la consistencia interna del análisis factorial exploratorio para buscar reducir el número de ítems, y se utilizó el coeficiente de fiabilidad alfa de Cronbach $(\alpha)$, y se siguieron las siguientes reglas generales son proporcionadas por George $\mathrm{y}$ Mallery (citado en Richer \& Bergeron, 2012) para el nivel de impacto.

\section{Resultados}

\section{Resultados de la Primera Fase}

Selección de Ítems. La tabla 1 contiene la contabilización de los comportamientos que hace un conductor agresivo, según respondieron los participantes de este primer estudio. Se 
separan las 21 respuestas con mayor frecuencia, y con ellas se construye el ICAT (ver Tabla 3). Las tres categorías que tuvieron mayor frecuencia fueron: (1) Agresiones verbales, (2) insultos y/o etiquetas, y (3) Claxon o bocina. Las tres categorías que tuvieron menor frecuencia fueron: (1) mala educación, (2) no respetar a las autoridades, y (3) responder agresivamente a las críticas.

Posteriormente se agruparon los ítems en base a las frecuencias más altas que indicaban uno de los aspectos considerados en el instrumento. Así se espera posicionar a los ítems dentro de los aspectos propuestos en el marco teórico. Algunos ítems que hicieron referencia al aspecto de poca consideración fueron: (1) bloquear el paso de otro vehículo, (2) invadir el flujo del tránsito agresivamente. Otros ítems que indicaron la presencia del aspecto de manifestación de frustración/enfado fueron: (1) tocar el claxon o bocina, comportarse irrespetuosa y/o discriminatoriamente. Algunos ítems que hicieron referencia al aspecto de conducción de riesgo fueron: no usar las direccionales antes de realizar una vuelta, conducir a exceso de velocidad. La colección completa de ítems se puede observar en la Tabla 4

\section{Resultados de la Segunda Fase}

Cribado de ítems. La mayoría de los ítems tuvo una media de entre 1.04 y 2.3, desviaciones estándar de entre .678 y 1.324 , asimetría de entre .594 y 3.43 , y curtosis de entre .473 y 13.23. El supuesto de normalidad fue violado en todos los ítems de acuerdo al test de Shapiro-Wilk $(p<.05)$. Se observa que los ítems son asimétricos hacia la izquierda, y aunque varía la intensidad, y la distribución es leptocúrtica. (ver Tabla 5)

Validez de Constructo. Una vez se seleccionaron los ítems, se evaluó la validez de constructo a través de un análisis factorial exploratorio. 
Análisis factorial exploratorio. Se llevó a cabo un análisis de componentes principales para evaluar los aspectos del comportamiento agresivo en los participantes de este estudio. Al inspeccionar la matriz de correlaciones, se comprobó que todos los ítems tenían al menos un coeficiente de correlación mayor a 0.3. La medida general de Kaiser-Meyer-Olkin (KMO) fue 0.91 con medidas de KMO individuales mayores a 0.7 La Prueba de Esfericidad de Bartlett fue estadísticamente significativa, $X^{2}=3303.45, g l=210, p \leq .001$, lo que indica que los datos son factorizables.

El análisis de componentes principales reveló cuatro componentes que explicaron el $35.92 \%, 8.28 \%, 7.10 \%$ y $5.83 \%$ de la varianza total, respectivamente. La inspección visual del gráfico de sedimentación indicó que se deberían retener tres factores (Cattell, 1966), Además, la solución de tres componentes cumplía con el criterio de interpretabilidad. Como tal, se mantuvieron tres componentes.

La solución de tres componentes explicó $60.77 \%$ de la varianza total. Se utilizó una rotación ortogonal tipo Varimax para facilitar la interpretación. La interpretación de los datos fue consistente con el comportamiento agresivo para lo que el Inventario fue diseñado, con altos pesos factoriales de ítems de manifestación de frustración/enfado en el primer componente, ítems de poca consideración en el segundo componente e ítems de conducción de riesgo en el tercer componente. Los pesos factoriales y comunalidades de la solución rotada se presentan en la Tabla 6.

Fiabilidad. Se evaluó la consistencia interna para evaluar la consistencia interna de los ítems que componen la escala propuesta. 
Consistencia interna. Para este estudio se obtuvo un alfa de Cronbach de .77, que según George y Mallery (citado en Richer \& Bergeron, 2012) y Vera-Jiménez, Ávila-Guerrero y Dorantes-Argandar (2014) es aceptable. Asimismo, se obtuvieron los alfa de Cronbach para los componentes de Manifestación de frustración/enfado, Poca Consideración y Conducción de Riesgo que fueron de .79, .67 y .49. De acuerdo a George y Mallery (citado en Richer \& Bergeron, 2012) fueron aceptable, cuestionable e inaceptable respectivamente.

\section{Resultados de la tercera fase}

Cribado de ítems. La mayoría de los ítems tuvo una media de entre 1.34 y 2.34 , desviaciones estándar de entre .79 y 1.39 , asimetría de entre .77 y 2.74 , y curtosis de entre -.69 y 7.68. El supuesto de normalidad fue violado en todos los ítems de acuerdo al test de Shapiro-Wilk $(p<.05)$. Se observa que los ítems son asimétricos hacia la izquierda, y aunque varía la intensidad, y la distribución es leptocúrtica. (ver Tabla 7)

Validez de Constructo. Se evaluó la consistencia de la validez de constructo a través de un segundo análisis factorial exploratorio.

Análisis factorial exploratorio. Se llevó a cabo un análisis de componentes principales para evaluar los aspectos del comportamiento agresivo en los participantes de este estudio. Fueron excluidos 99 participantes por usar el mismo tipo de respuesta en toda la escala. Asimismo, no fueron considerados en el análisis 148 participantes por contar con una distancia Malahanobis mayor al valor crítico de 46.8. Por lo que se analizaron los datos en 1522 participantes.Al inspeccionar la matriz de correlaciones, se comprobó que todos los ítems tenían al menos un coeficiente de correlación mayor a 0.3. La medida general de Kaiser-Meyer-Olkin (KMO) fue 0.96 
con medidas de KMO individuales mayores a 0.8 , clasificaciones de "meritorias" a "maravillosas" según Kaiser (1974).La Prueba de Esfericidad de Bartlett fue estadísticamente significativa, $X^{2}$ $=15657.16, g l=210, p \leq .001$, lo que indica que los datos son factorizables.

El análisis de componentes principales reveló cuatro componentes que explicaron el $43.21 \%, 6.89 \%, 6.18 \%$ y $4.99 \%$ de la varianza total, respectivamente. La inspección visual del gráfico de sedimentación indicó que se deberían retener tres factores (Cattell, 1966), Además, la solución de tres componentes cumplía con el criterio de interpretabilidad. Como tal, se mantuvieron tres componentes.

La solución de tres componentes explicó $64.09 \%$ de la varianza total. Se utilizó una rotación ortogonal tipo Varimax para facilitar la interpretación. La interpretación de los datos fue consistente con el comportamiento agresivo para lo que el Inventario fue diseñado, con altos pesos factoriales de ítems de manifestación de frustración/enfado en el primer componente, ítems de poca consideración en el segundo componente e ítems de conducción de riesgo en el tercer componente. Los pesos factoriales y comunalidades de la solución rotada se presentan en la Tabla 8.

Consistencia interna. Para este estudio se obtuvo un alfa de Cronbach de .88, que según George y Mallery (citado en Richer \& Bergeron, 2012) y Vera-Jiménez, Ávila-Guerrero y Dorantes-Argandar (2014) es bueno. Asimismo, se obtuvieron los alfa de Cronbach para los componentes de Manifestación de frustración/enfado, Poca Consideración y Conducción de Riesgo que fueron de $.89, .70$ y .60. De acuerdo a George y Mallery (citado en Richer \& Bergeron, 2012) fueron bueno, aceptable y cuestionable respectivamente. 


\section{Discusión}

La caracterización del conductor agresivo es indispensable para la resolución de la problemática social que se está padeciendo en la ciudad de Cuernavaca al respecto de la agresividad y accidentalidad vial (Dorantes-Argandar et al., 2015a, 2015b), y en gran parte del país. Muchos de los estudios realizados en otros países empiezan a perfilar al conductor agresivo como un elemento de necesaria intervención (Abdu et al., 2012; Bachoo et al., 2013; Bailey et al., 2016; Balogun, Shenge, y Oladipo, 2012; Berdoulat et al., 2013; Brewer, 2000; Cervantes-Trejo, 2009; Constantinou et al., 2011; Cuevas Colunga et al., 2014), gracias a las herramientas que se han desarrollado en el extranjero (Bener et al.,2008; Clapp et al., 2011, 2014; Deffenbacher et al., 1996; Deffenbacher, Deffenbacher et al., 2003; Dula \& Geller, 2003).

El propósito fundamental de la primera fase fue obtener el contenido con el que se pretendería evaluar los comportamientos agresivos que se realizan en el tránsito. La categorización de los ítems es consistente con lo reportado en estudios anteriores. Por ejemplo, el uso del claxon y gritar se incluyó en la dimensión Manifestación de la Frustración, lo cual es consistente con Deffenbacher, Deffenbacher et al. (2003) el cual los incluye en los comportamientos que manifiestan el enfado. Es posible que la técnica utilizada para la generación de los ítems no haya sido la más adecuada, por lo que el contenido de los ítems no es necesariamente idéntico al de otros constructos teóricos de la misma. Se recomienda una nueva realización del contenido de la escala para asegurarse que la estructuración del fenómeno es verdaderamente así, o si estos resultados se deben a algún error metodológico.

El proceso de la selección de los ítems fue exhaustivo. Los ítems parecen representar el fenómeno de la agresividad vial de una manera que refleja la realidad local, y tiene cierta semejanza con los constructos encontrados en otros países. Sin embargo, es necesario precisar si 
los ítems incluidos en este estudio que no coinciden con otras escalas se deben a que este estudio sufre de errores metodológicos o si la realidad local es en extremo diferente como para ameritar un encuadre muy ajustado a sus particularidades. Esto incluye lo ítems sobre los comentarios discriminatorios e impactar el vehículo a manera de agresión.

Durante la segunda fase se observó un adecuado comportamiento de los análisis estadísticos, aunque la muestra no fue lo suficientemente robusta y no se obtuvieron criterios con los cuales contrastar los resultados obtenidos. El constructo parece ser aceptable y la alineación de los aspectos es un poco diferente a la observada en la literatura revisada (Deffenbacher et al., 2002; Fruhen \& Flin, 2015; Harris et al., 2014; Kovácsová et al., 2014; Li et al., 2014; Richer \& Bergeron, 2012; Sârbescu et al., 2014; Stephens \& Sullman, 2014; Taubman - Ben-Ari \& Skvirsky, 2016) a excepción del modelo teórico propuesto por Shinar (2007).Se esperaba un mejor resultado, dado que hubo problemas en (1) las correlaciones entre los ítems lo que puede significar problemas de validez factorial convergente; (2) correlaciones de los ítems con un componente que teóricamente hablando no debería de ser, lo que significaría problemas de validez factorial convergente; y (3) la existencia de ítems con cargas cruzadas, lo que puede significar problemas de validez factorial convergente y divergente. Esto supuso ajustar el número de ítems para encontrar la solución final de componentes principales sin que se tuviera que excluir demasiados ítems. Se excluyeron 10 ítems en la solución final de componentes, lo cual significa un gran número de elementos. Esto se puede deber a la problemática de validez factorial convergente y divergente. Un ítem excluido del componente Poca Consideración, Invadir el flujo del tránsito agresivamente, presentó problemas de cargas cruzadas, lo cual causó afectaciones en la validez factorial convergente y divergente. Sin embargo, el ítem si es consistente con el modelo teórico propuesto por Shinar (2007). Este pudo ser ocasionado por la falta de evaluación de la validez de 
contenido (Cohen \& Swerdlik, 2010). Sin embargo, la distribución factorial de los ítems coincide con la postura teórica de Shinar (2007). El instrumento muestra buenos niveles de fiabilidad en base a los estadísticos generados para la consistencia interna, cuyos valores fueron de nivel aceptable (Vera-Jiménez et al., 2014). Posiblemente no fue excelente debido a que el número de ítems se vio reducido y las correlaciones entre los ítems no fueron tan altas como se esperaría.

El tercer estudio muestra una estructura de 3 factores, los cual coincide con el modelo teórico de comportamiento agresivo en el tránsito de Shinar (2007). La muestra superior del tercer estudio permite observar una estructura más sólida en el número de ítems para cada factor. El estudio tuvo diversas dificultades metodológicas que deberían de ser abordadas en futuros estudios. Los datos que a través del ICAT se recauden en dicha localidad serán fiables sólo en consideración de los resultados que se generaron en este estudio. El constructo generado es de mayor solidez en la tercera fase. Se explica un amplio nivel de la varianza y los factores obtienen alfas de excelente nivel. Lamentablemente, los aspectos del constructo no se alinean con la literatura consultada y el análisis realizado en la segunda fase de este estudio, y se asemejan solamente al modelo teórico propuesto por Shinar (2007).Al igual que en el segundo estudio, es posible que el no haber usado otras técnicas para evaluar la validez de constructo (Cohen \& Swerdlik, 2010) ocasionara dificultades en la validez convergentes y divergentes en la agrupación factorial de los ítems.

La evaluación de la fiabilidad en la tercera fase fue de mayor amplitud, y se encontraron resultados de buen nivel. Los índices de fiabilidad son mejores a los encontrados en el segundo estudio y más similares a los encontrados durante el proceso de la validación de los instrumentos de Deffenbacher para España (Herrero-Fernández, 2011a; 2011b) y en otros países (Ge et al., 2015; 
Gras et al., 2015; Sârbescu, 2012; Sârbescu, Stanojević, \& Jovanović, 2014; Stephens \& Sullman, 2014).La consistencia interna de la solución

factorial de la escala en la tercera fase fue excelente. Empero, la de las dimensiones varió entre aceptable y excelente. Esto nos permite concluir que los resultados obtenidos a través de esta escala son fiables. Sin embargo, la replicación de este estudio debería de considerar abordar las problemáticas metodológicas observadas durante la generación de los ítems para asegurarse obtener mejores niveles en la fase de validez factorial.

\section{Conclusiones}

La validación local del Inventario de Comportamientos Agresivos en el Tránsito es aceptable. Hubo algunas dificultades con las agrupaciones factoriales debido a que no se siguió un proceso comprehensivo para la construcción y validación del instrumento. Sin embargo, se considera que la herramienta es útil. Ahora, con esta herramienta, se puede realizar la caracterización del conductor agresivo cuernavacense, y determinar cuáles son los factores psicosociales, psicológicos, de personalidad y culturales que están inherentes en la problemática de la agresividad vial. Se presenta un instrumento con buenos indicadores estadísticos que permitirán evaluar la agresividad en la movilidad de una manera precisa.

La validación del ICAT permitirá realizar futuras investigaciones que permitirán conocer tanto los factores de personalidad que subyacen a la agresividad vial en la ciudad de Cuernavaca, así como los factores psicosociales y contextuales que la promueven. Esto permitirá realizar acciones que permitan reducir los niveles de agresividad al volante, lo que a su vez deberá de resultar en una mejora en el bienestar de la comunidad, así como en la seguridad vial misma.

\section{Limitaciones}


Los resultados de este estudio se limitan exclusivamente a la ciudad de Cuernavaca, para poder realizar estudios regionales o nacionales es preciso contar con la validación a un nivel mucho más grande.

\section{Sugerencias a futuras investigaciones}

Se recomienda realizar un estudio longitudinal para abonar a la validación del instrumento, así como aplicarlo con otras medidas que aporten evidencias de validez externa al instrumento. Adicionalmente, es preciso realizar la replicabilidad del estudio para confirmar la asignación factorial de los ítems.

\section{Referencias}

Abdu, R., Shinar, D., \& Meiran, N. (2012). Situational (state) anger and driving. Transportation Research Part F: Traffic Psychology and Behaviour, 15(5), 575-580. http://doi.org/10.1016/j.trf.2012.05.007

Al Reesi, H., Al Maniri, A., Plankermann, K., Al Hinai, M., Al Adawi, S., Davey, J., \&Freeman, J. (2013). Risky driving behavior among university students and staff in the Sultanate of Oman. Accident Analysis \& Prevention, 58, 1-9. http://doi.org/10.1016/j.aap.2013.04.021

Amado, S., Arıkan, E., Kaça, G., Koyuncu, M., \& Turkan, B. N. (2014). How accurately do drivers evaluate their own driving behavior? An on-road observational study. Accident Analysis \& Prevention, 63, 65-73. http://doi.org/10.1016/j.aap.2013.10.022

Arnau-Sabatés, L., Sala-Roca, J., \& Jariot-Garcia, M. (2012). Emotional abilities as predictors of risky driving behavior among a cohort of middle aged drivers. Accident Analysis \& Prevention (Vol. 45). 
Awopetu Ronke, G., \& Igbo Happiness, I. (2015). An Assessment of Aggressive Behaviour between Prison Inmates and Non-prison Inmates in Makurdi Metropolis, Nigeria. Procedia Social and Behavioral Sciences, 190, 502-509. http://doi.org/10.1016/j.sbspro.2015.05.034

Ba, Y., Zhang, W., Salvendy, G., Cheng, A. S. K., \& Ventsislavova, P. (2016). Assessments of risky driving: A Go/No-Go simulator driving task to evaluate risky decision-making and associated behavioral patterns. Applied Ergonomics, 52, 265-274. http://doi.org/10.1016/j.apergo.2015.07.020

Bachoo, S., Bhagwanjee, A., \& Govender, K. (2013). The influence of anger, impulsivity, sensation seeking and driver attitudes on risky driving behaviour among post-graduate university students in Durban, South Africa. Accident Analysis \& Prevention, 55, 67-76. http://doi.org/10.1016/j.aap.2013.02.021

Bailey, S., Lennon, A., \& Watson, B. (2016). Getting mad may not mean getting even: The influence of drivers' ethical ideologies on driving anger and related behaviour. Transportation Research Part F: Traffic Psychology and Behaviour, 36, 104-116. http://doi.org/10.1016/j.trf.2015.11.004

Baker, A. S., Litwack, S. D., Clapp, J. D., Gayle Beck, J., \& Sloan, D. M. (2014). The Driving Behavior Survey as a Measure of Behavioral Stress Responses to MVA-Related PTSD. Behavior Therapy. http://doi.org/10.1016/j.beth.2014.02.005

Balogun, S. K., Shenge, N. A., \& Oladipo, S. E. (2012). Psychosocial factors influencing aggressive driving among commercial and private automobile drivers in Lagos metropolis. The Social Science Journal, 49(1), 83-89. http://doi.org/10.1016/j.soscij.2011.07.004 
Bassett, J. F., Cate, K. L., \& Dabbs, J. M. J. (2002). Individual differences in self-presentation style: Driving an automobile and meeting a stranger. Self and Identity, 1(3), 281-288. http://doi.org/10.1080/152988602760124892

Battistich, V., \& Hom, A. (1997). The Relationship between Sutdents' Sense of Their School as a Community and Their Involvement in Problem Behaviors. American Journal of Public Health, 87(12), 1997-2001. http://doi.org/10.2105/AJPH.87.12.1997

Beanland, V., Sellbom, M., \& Johnson, A. K. (2014). Personality domains and traits that predict self-reported aberrant driving behaviours in a southeastern US university sample. Accident Analysis \& Prevention, 72, 184-192. http://doi.org/10.1016/j.aap.2014.06.023

Beck, K. H., Ali, B., \& Daughters, S. B. (2014). Distress Tolerance as a Predictor of Risky and $\begin{array}{lllll}\text { Aggressive } & \text { Driving. }\end{array}$ http://doi.org/10.1080/15389588.2013.829569

Beck, K. H., Daughters, S. B., \& Ali, B. (2013). Hurried driving: Relationship to distress tolerance, driver anger, aggressive and risky driving in college students. Accident Analysis \& Prevention, 51, 51-55. http://doi.org/10.1016/j.aap.2012.10.012

Beck, K. H., Wang, M. Q., \& Mitchell, M. M. (2006). Concerns, dispositions and behaviors of aggressive drivers: What do self-identified aggressive drivers believe about traffic safety? Journal of Safety Research, 37(2), 159-165. http://doi.org/10.1016/j.jsr.2006.01.002

Beck, K. H., \& Watters, S. (2016). Characteristics of college students who text while driving: Do their perceptions of a significant other influence their decisions? Transportation Research Part F: Traffic Psychology and Behaviour, 37, 119-128. 
Bener, A., Özkan, T., \& Lajunen, T. (2008). The Driver Behaviour Questionnaire in Arab Gulf countries: Qatar and United Arab Emirates. Accident Analysis \& Prevention, 40(4), 14111417. http://doi.org/10.1016/j.aap.2008.03.003

Berdoulat, E., Vavassori, D., \& Sastre, M. T. M. (2013). Driving anger, emotional and instrumental aggressiveness, and impulsiveness in the prediction of aggressive and transgressive driving. Accident Analysis \& Prevention, 50, 758-767. http://doi.org/10.1016/j.aap.2012.06.029

Björklund, G. M. (2008). Driver irritation and aggressive behaviour. Accident Analysis \& Prevention, 40(3), 1069-1077. http://doi.org/10.1016/j.aap.2007.10.014

Blankenship, K. L., \& Nesbit, S. M. (2013). Driving stimuli increases accessibility of aggressionrelated concepts in "angry" drivers. Personality and Individual Differences, 55(2), 135-140. http://doi.org/10.1016/j.paid.2013.02.014

Bonus, J. A., Peebles, A., \& Riddle, K. (2015). The influence of violent video game enjoyment on hostile attributions. Computers in Human Behavior, 52, 472-483. http://doi.org/10.1016/j.chb.2015.05.044

Brewer, A. M. (2000). Road rage: What, who, when, where and how? Transport Reviews, 20(1). http://doi.org/http://dx.doi.org/10.1080/014416400295338

Britt, T. W., \& Garrity, M. J. (2006). Attributions and personality as predictors of the road rage response. The British Journal of Social Psychology / the British Psychological Society, 45( $\mathrm{Pt}$ 1), 127-147. http://doi.org/10.1348/014466605X41355

Castellani, V., Pastorelli, C., Eisenberg, N., Caffo, E., Forresi, B., \& Gerbino, M. (2014). The 
development of perceived maternal hostile, aggressive conflict from adolescence to early adulthood: Antecedents and outcomes. Journal of Adolescence, 37(8), 1517-1527. http://doi.org/10.1016/j.adolescence.2014.07.001

Cattell, R. B. (1966). The scree test for the number of factors. Multivariate Behavioral Research, $1(2), 11-21$.

Cervantes-Trejo, A. (2009). Choques dejan heridos fuera de registro oficial. El Universal. Available online: http://www.eluniversal.com.mx/nacion/171035.html Access: 29-062015.

Clapp, J. D., Baker, A. S., Litwack, S. D., Sloan, D. M., \& Beck, J. G. (2014). Properties of the Driving Behavior Survey among individuals with motor vehicle accident-related posttraumatic stress disorder. Journal of Anxiety Disorders, 28(1), 1-7. http://doi.org/10.1016/j.janxdis.2013.10.008

Clapp, J. D., Olsen, S. A., Beck, J. G., Palyo, S. A., Grant, D. M., Gudmundsdottir, B., \& Marques, L. (2011). The Driving Behavior Survey: Scale construction and validation. Journal of Anxiety Disorders, 25(1), 96-105. http://doi.org/10.1016/j.janxdis.2010.08.008

Cohen, R. J., \& Swerdlik, M. E. (2010). Psychological Testing and Assessment: an Introduction to Tests and Measurement. USA: McGraw-Hill.

Constantinou, E., Panayiotou, G., Konstantinou, N., Loutsiou-Ladd, A., \& Kapardis, A. (2011). Risky and aggressive driving in young adults: Personality matters. Accident Analysis \& Prevention, 43(4), 1323-1331. http://doi.org/10.1016/j.aap.2011.02.002

Cuevas Colunga, A. C., Villegas Villegas, N., Mayoral Grajeda, E. F., \& Mendoza Díaz, A. (2014). 
Anuario estadístico de accidentes en carreteras federales (2012), (57), 98. Retrieved from http://207.248.177.30/mir/uploadtests/32470.177.59.1.02 Anuario accidentes 2012.pdf

Deffenbacher, J. L. (2011). Cognitive-Behavioral Conceptualization and Treatment of Anger. Cognitive and Behavioral Practice, 18(2), 212-221. http://doi.org/10.1016/j.cbpra.2009.12.004

Deffenbacher, J. L. (2016). A review of interventions for the reduction of driving anger. Transportation Research Part F: Traffic Psychology and Behaviour. http://doi.org/10.1016/j.trf.2015.10.024

Deffenbacher, J. L., Deffenbacher, D. M., Lynch, R. S., \& Richards, T. L. (2003). Anger, aggression, and risky behavior: a comparison of high and low anger drivers. Behaviour Research and Therapy, 41(6), 701-718. http://doi.org/10.1016/S0005-7967(02)00046-3

Deffenbacher, J. L., Lynch, R. S., Filetti, L. B., Dahlen, E. R., \& Oetting, E. R. (2003). Anger, aggression, risky behavior, and crash-related outcomes in three groups of drivers. Behaviour Research and Therapy, 41(3), 333-349. http://doi.org/10.1016/S0005-7967(02)00014-1

Deffenbacher, J. L., Lynch, R. S., Oetting, E. R., \& Swaim, R. C. (2002). The Driving Anger Expression Inventory: a measure of how people express their anger on the road. Behaviour Research and Therapy, 40(6), 717-737. http://doi.org/10.1016/S0005-7967(01)00063-8

Deffenbacher, J. L., Oetting, E. R., Lynch, R. S., \& Morris, C. D. (1996). The expression of anger and its consequences. Behaviour Research and Therapy, 34(7), 575-590. http://doi.org/10.1016/0005-7967(96)00018-6

Deffenbacher, J. L., Petrilli, R. T., Lynch, R. S., Oetting, E. R., \& Swaim, R. C. (2003). The 
driver's angry thoughts questionnaire: A measure of angry cognitions when driving. Cognitive Therapy and Research, 27(4), 383-402. http://doi.org/10.1023/A:1025403712897

Dorantes-Argandar, G., Cerda-Macedo, E. A., Tortosa-Gil, F., \& Ferrero Berlanga, J. (2015a). Accidentalidad de automóviles de uso particular en méxico: influencia del estrés y la agresividad. Psiencia Revista Latinoamericana de Ciencia Psicologica, (7), 418-427. http://doi.org/10.5872/psiencia/7.3.121

Dorantes-Argandar, G., Cerda-Macedo, E. A., Tortosa-Gil, F., \& Ferrero Berlanga, J. (2015b). Agresividad vial como predictor del estrés y del comportamiento prosocial, y su influencia en la siniestralidad de vehículos particulares en méxico. Ansiedad Y Estrés, 21(3), 207-217.

Dorantes-Argandar, G., \& Ferrero-Berlanga, J. (2017). Impulsivity and Aggressive Driving as mediators between Self-Esteem and Stress in Mexican drivers. Journal of Psychology and Behavioral Science, 4(2), 17-29.

Dorantes-Argandar, G., Tortosa-Gil, F., \& Ferrero-Berlanga, J. (2016). Measuring situations that stress Mexicans while driving. Transportation Research Part F: Traffic Psychology and Behaviour, 37, 154-161. http://doi.org/10.1016/j.trf.2015.12.014

Dula, C. S., \& Geller, E. S. (2003). Risky, aggressive, or emotional driving: Addressing the need for consistent communication in research. Journal of Safety Research, 34(5), 559-566. http://doi.org/10.1016/j.jsr.2003.03.004

Fruhen, L. S., \& Flin, R. (2015). Car driver attitudes, perceptions of social norms and aggressive driving behaviour towards cyclists. Accident Analysis \& Prevention, 83, 162-170. http://doi.org/10.1016/j.aap.2015.07.003 
Ge, Y., Qu, W., Zhang, Q., Zhao, W., \& Zhang, K. (2015). Psychometric adaptation of the driving anger expression inventory in a Chinese sample. Transportation Research Part F: Traffic Psychology and Behaviour, 33, 75-86. http://doi.org/10.1016/j.trf.2015.07.008

Gidron, Y., Gaygisız, E., \& Lajunen, T. (2014). Hostility, driving anger, and dangerous driving: The emerging role of hemispheric preference. Accident Analysis \& Prevention, 73, 236-241. http://doi.org/10.1016/j.aap.2014.09.011

Gras, M.-E., Font-Mayolas, S., Patiño, J., Baltasar, A., Planes, M., \& Sullman, M. J. M. (2015). Resilience and the expression of driving anger. Transportation Research Part F: Traffic Psychology and Behaviour. http://doi.org/10.1016/j.trf.2015.09.005

Hamdar, S. H., Qin, L., \& Talebpour, A. (2016). Weather and road geometry impact on longitudinal driving behavior: Exploratory analysis using an empirically supported acceleration modeling framework. Transportation Research Part C: Emerging Technologies, 67, 193-213. http://doi.org/10.1016/j.trc.2016.01.017

Harris, P. B., Houston, J. M., Vazquez, J. A., Smither, J. A., Harms, A., Dahlke, J. A., \& Sachau, D. A. (2014). The Prosocial and Aggressive Driving Inventory (PADI): A self-report measure of safe and unsafe driving behaviors. Accident Analysis and Prevention, 72, 1-8. http://doi.org/10.1016/j.aap.2014.05.023

Helfritz-Sinville, L. E., \& Stanford, M. S. (2014). Hostile attribution bias in impulsive and premeditated aggression. Personality and Individual Differences, 56(1), 45-50. http://doi.org/10.1016/j.paid.2013.08.017

Hennessy, D. a., \& Wiesenthal, D. I. (2002). Aggression, violence, and vengence among male and 
female drivers.

Transportant

Quarterly.

Retrieved

from

http://web.a.ebscohost.com.ezproxy.lib.swin.edu.au/ehost/pdfviewer/pdfviewer?sid=ef5761

3c-39c6-449e-82f8-1418b95452a6@sessionmgr4005\&vid=2\&hid=4114

Herrero-Fernández, D. (2011a). Psychometric adaptation of the driving anger expression inventory in a spanish sample: Differences by age and gender. Transportation Research Part F: Traffic Psychology and Behaviour, 14, 324-329. http://doi.org/10.1016/j.trf.2011.03.001

Herrero-Fernández, D. (2011b). Psychometric adaptation of the Driving Anger Expression Inventory in a Spanish sample: Differences by age and gender. Transportation Research Part F: Traffic Psychology and Behaviour, 14(4), 324-329. http://doi.org/10.1016/j.trf.2011.03.001

Hinojosa-Reyes, R., Jiménez-Sánchez, P. L., Hernández-Hernández, V., \& Campos Alanís, J. (2012). SINIESTRALIDAD POR ACCIDENTES DE TRÁNSITO EN MÉXICO: UNA APROXIMACIÓN DESDE EL ANÁLISIS ESPACIAL. Geografía Y Sistemas de Información Geográfica, 4(4), 291-309.

Houstoa, J. M., Harris, P. B., \& Norman, M. (2003). The Aggressive Driving Behavior Scale: Developing a Self-Report Measure of Unsafe Driving Practices. North American Journal of Psychology, 5(2), 269-279.

Hsieh, H.-F., Heinze, J. E., Aiyer, S. M., Stoddard, S. A., Wang, J.-L., \& Zimmerman, M. A. (2015). Cross-domain influences on youth risky driving behaviors: A developmental cascade analysis. Journal of Applied Developmental Psychology, 38, 11-21. http://doi.org/10.1016/j.appdev.2015.03.002 
Instituto Nacional de Estadistica y Geografía. (2010). Principales resultados del Censo de Población y Vivienda. Morelos. Instituto Nacional de Estadística y Geografía.

Kovácsová, N., Lajunen, T., \& Rošková, E. (2016). Aggression on the road: Relationships between dysfunctional impulsivity, forgiveness, negative emotions, and aggressive driving. Transportation Research Part F: Traffic Psychology and Behaviour, 42, 286-298. http://doi.org/10.1016/j.trf.2016.02.010

Kovácsová, N., Rošková, E., \& Lajunen, T. (2014). Forgivingness, anger, and hostility in aggressive driving. Accident Analysis \& Prevention, 62, 303-308. http://doi.org/10.1016/j.aap.2013.10.017

Lajunen, T., \& Parker, D. (2001). Are aggressive people aggressive drivers? A study of the relationship between self-reported general aggressiveness, driver anger and aggressive driving. Accident Analysis \& Prevention, 33(2), 243-255. http://doi.org/10.1016/S00014575(00)00039-7

Lawrence, C., \& Hodgkins, E. (2009). Personality influences on interpretations of aggressive behavior: The role of provocation sensitivity and trait aggression. Personality and Individual Differences, 46(3), 319-324. http://doi.org/10.1016/j.paid.2008.10.022

Lazarus, R., \& Folkman, S. (1984). Stress, appraisal, and coping. Behaviour Research and Therapy (Vol. 23). New York: Springer Publishing Company. http://doi.org/10.1016/00057967(85)90087-7

Li, F., Yao, X., Jiang, L., \& Li, Y. (2014). Driving anger in China: Psychometric properties of the Driving Anger Scale (DAS) and its relationship with aggressive driving. Personality and 
Individual Differences, 68, 130-135. http://doi.org/10.1016/j.paid.2014.04.018

Lima-Aranzaes, C. C., Juárez-García, A. \& Arias-Galicia, F. (2012). Un estudio exploratorio sobre estresores laborales en conductores de transporte público colectivo en el estado de Morelos, México. En Arias Galicia, F. y Juárez García, A. (Eds.). Agotamiento profesional y estrés: Hallazgos desde México y otros países latinoamericanos. México: Miguel Ángel Porrúa.

Locke, K. D. (2009). Aggression, narcissism, self-esteem, and the attribution of desirable and humanizing traits to self versus others. Journal of Research in Personality, 43(1), 99-102. http://doi.org/10.1016/j.jrp.2008.10.003

Martins, N. (2013). Televised relational and physical aggression and children's hostile intent attributions. Journal of Experimental Child Psychology, 116(4), 945-952. http://doi.org/10.1016/j.jecp.2013.05.006

Observatorio Nacional de Lesiones. (2013). Perfil Estatal Morelos. (Observatorio Nacional de Lesiones, Ed.). Retrieved from http://www.conapra.salud.gob.mx/Interior/Documentos/Observatorio/Perfiles/17_Morelos.p df

Oviedo-Trespalacios, O., Haque, M. M., King, M., \& Washington, S. (2016). Understanding the impacts of mobile phone distraction on driving performance: A systematic review. Transportation Research Part C: Emerging Technologies, 72, 360-380. http://doi.org/10.1016/j.trc.2016.10.006

Özkan, T., Lajunen, T., Chliaoutakis, J. E., Parker, D., \& Summala, H. (2006). Cross-cultural differences in driving skills: A comparison of six countries. Accident Analysis \& Prevention, 38(5), 1011-1018. http://doi.org/10.1016/j.aap.2006.04.006 
Parsian, N., \& Dunning, T. (2009). Developing and validating a questionnaire to measure spirituality: A psychometric process. Global Journal of Health Science, 1(1), 2-11. http://doi.org/10.5539/gjhs.v1n1p2

Pawliczek, C. M., Derntl, B., Kellermann, T., Gur, R. C., Schneider, F., \& Habel, U. (2013). Anger under Control: Neural Correlates of Frustration as a Function of Trait Aggression. PLoS ONE, 8(10), 1-11. http://doi.org/10.1371/journal.pone.0078503

Pearson, M. R., Murphy, E. M., \& Doane, A. N. (2013). Impulsivity-like traits and risky driving behaviors among college students. Accident Analysis \& Prevention, 53, 142-148. http://doi.org/10.1016/j.aap.2013.01.009

Poó, F. M. (2014). Evaluación Multidimensional del Estilo de Conducción. Su Relación con Variables Psicológicas y Comprotamientos de Riesgo. Tesis Doctoral Universidad Nacional de Mar del Plata. Argentina.

Poythress, N. G., \& Hall, J. R. (2011). Psychopathy and impulsivity reconsidered. Aggression and Violent Behavior, 16(2), 120-134. http://doi.org/10.1016/j.avb.2011.02.003

Przepiorka, A. M., Blachnio, A., \& Wiesenthal, D. L. (2014). The determinants of driving aggression among Polish drivers. Transportation Research Part F: Traffic Psychology and Behaviour, 27, 69-80. http://doi.org/10.1016/j.trf.2014.09.007

Richer, I., \& Bergeron, J. (2012). Differentiating risky and aggressive driving: Further support of the internal validity of the Dula Dangerous Driving Index. Accident Analysis \& Prevention, 45, 620-627. http://doi.org/10.1016/j.aap.2011.09.014

Rodríguez, R. A., Virguez, E. A., Rodríguez, P. A., \& Behrentz, E. (2016). Influence of driving 
patterns on vehicle emissions: A case study for Latin American cities. Transportation Research Part D: Transport and Environment, 43, 192-206. http://doi.org/10.1016/j.trd.2015.12.008

Rosenbloom, T., \& Wultz, B. (2011). Thirty-day self-reported risky driving behaviors of ADHD and non-ADHD drivers. Accident Analysis \& Prevention, 43(1), 128-133. http://doi.org/10.1016/j.aap.2010.08.002

Sârbescu, P. (2012). Aggressive driving in Romania: Psychometric properties of the Driving Anger Expression Inventory. Transportation Research Part F: Traffic Psychology and Behaviour, 15(5), 556-564. http://doi.org/10.1016/j.trf.2012.05.009

Sârbescu, P., Stanojević, P., \& Jovanović, D. (2014). A cross-cultural analysis of aggressive driving: Evidence from Serbia and Romania. Transportation Research Part F: Traffic Psychology and Behaviour, 24, 210-217. http://doi.org/10.1016/j.trf.2014.04.002

Seligman, M. E. P. (2002). Positive Psychology, Positive Prevention, and Positive Therapy. In C. R. Snyder \& J. L. Shane (Eds.), Handbook of Positive Psychology (1st ed., pp. 3-12). New York: Oxford University Press.

Shinar, D. (2007). Traffic Safety and Human Behavior (1st ed.). Emerald Group Publishing Limited.

Smith, P., Waterman, M., \& Ward, N. (2006). Driving aggression in forensic and non-forensic populations: relationships to self-reported levels of aggression, anger and impulsivity. Bri J Psychol, 97(Pt 3), 387-403. http://doi.org/10.1348/000712605X79111

Stephens, A. N., \& Groeger, J. A. (2009). Situational specificity of trait influences on drivers' 
evaluations and driving behaviour. Transportation Research Part F: Traffic Psychology and Behaviour, 12(1), 29-39. http://doi.org/10.1016/j.trf.2008.06.005

Stephens, A. N., Hill, T., \& Sullman, M. J. M. (2016). Driving anger in Ukraine: Appraisals, not trait driving anger, predict anger intensity while driving. Accident Analysis \& Prevention, 88 , 20-28. http://doi.org/10.1016/j.aap.2015.11.017

Stephens, A. N., \& Sullman, M. J. M. (2014). Development of a short form of the driving anger expression inventory. Accident Analysis \& Prevention, 72, 169-176. http://doi.org/10.1016/j.aap.2014.06.021

Strandell, J. (2016). Culture, cognition and behavior in the pursuit of self-esteem. Poetics, 54, 14 24. http://doi.org/10.1016/j.poetic.2015.08.007

Sullman, M. J. M., \& Stephens, A. N. (2013). A comparison of the Driving Anger Scale and the Propensity for Angry Driving Scale. Accident Analysis \& Prevention, 58, 88-96. http://doi.org/10.1016/j.aap.2013.05.002

Sullman, M. J. M., Stephens, A. N., \& Yong, M. (2014). Driving anger in Malaysia. Accident Analysis \& Prevention, 71, 1-9. http://doi.org/10.1016/j.aap.2014.04.019

Taubman - Ben-Ari, O., \& Skvirsky, V. (2016). The multidimensional driving style inventory a decade later: Review of the literature and re-evaluation of the scale. Accident Analysis \& Prevention, 93, 179-188. http://doi.org/10.1016/j.aap.2016.04.038

Treviño-Siller, S., Híjar, M. \& Mora, G. (2011). Priorisation of road traffic injury interventions: results of a participative research with stakeholders in México. International Journal of Injury Control and Safety Promotion. 18(3), 219-225. 
Vanlaar, W., Simpson, H., Mayhew, D., \& Robertson, R. (2008). Aggressive driving: A survey of attitudes, opinions and behaviors. Journal of Safety Research, 39(4), 375-381. http://doi.org/10.1016/j.jsr.2008.05.005

Vera-Jiménez, J. A., Ávila-Guerrero, M. E., \& Dorantes-Argandar, G. (2014). Manual de evaluación de la victimización y percepción de la violencia, delincuencia, e inseguridad y la confianza en las instituciones (1st ed.). Cuernavaca: Editorial UAEM. Retrieved from http://libros.uaem.mx/?product=manual-de-evaluacion-de-la-victimizacion-y-percepcionde-la-violencia-delincuencia-e-inseguridad-y-la-confianza-en-las-instituciones

Villieux, A., \& Delhomme, P. (2010). Driving anger and its expressions: Further evidence of validity and reliability for the Driving Anger Expression Inventory French adaptation. Journal of Safety Research, 41(5), 417-422. http://doi.org/10.1016/j.jsr.2010.08.003

Walitzer, K. S., Deffenbacher, J. L., \& Shyhalla, K. (2015). Alcohol-Adapted Anger Management Treatment: A Randomized Controlled Trial of an Innovative Therapy for Alcohol Dependence. Journal of Substance Abuse Treatment, 59, 83-93. http://doi.org/10.1016/j.jsat.2015.08.003

Werner, N. E. (2012). Do hostile attribution biases in children and parents predict relationally aggressive behavior? The Journal of Genetic Psychology, 173(3), 221-45. http://doi.org/10.1080/00221325.2011.600357

Willemsen, J., Dula, C. S., Declercq, F., \& Verhaeghe, P. (2008). The Dula Dangerous Driving Index: An investigation of reliability and validity across cultures. Accident Analysis \& Prevention, 40(2), 798-806. http://doi.org/10.1016/j.aap.2007.09.019 
Wu, L. Z., Zhang, H., Chiu, R. K., Kwan, H. K., \& He, X. (2014). Hostile Attribution Bias and Negative Reciprocity Beliefs Exacerbate Incivility’s Effects on Interpersonal Deviance. Journal of Business Ethics, 120(2), 189-199. http://doi.org/10.1007/s10551-013-1658-6

Zhang, T., Chan, A. H. S., \& Zhang, W. (2015). Dimensions of driving anger and their relationships with aberrant driving. Accident Analysis \& Prevention, 81, 124-133. http://doi.org/10.1016/j.aap.2015.05.005

Zillmann, D., \& Weaver, J. B. (2007). Aggressive personality traits in the effects of violent imagery on unprovoked impulsive aggression. Journal of Research in Personality, 41(4), 753-771. http://doi.org/10.1016/j.jrp.2006.08.006 


\section{Apéndice}

\section{Tabla 1}

Revisión de Estudios que Abordan el Tema de la Agresión por Ámbitos

\begin{tabular}{ll}
\hline Ámbito & Temática \\
\hline Personalidad & Influencia de la personalidad en la interpretación del comportamiento agresivo \\
& (Lawrence \& Hodgkins, 2009) \\
& Rasgos de personalidad agresiva (Zillmann \& Weaver, 2007) \\
Actividad & Correlaciones neuronales entre la frustración y la agresión de rasgo (Pawliczek et \\
cerebral & al., 2013) \\
Impulsividad & Psicopatía e impulsividad (Poythress \& Hall, 2011) \\
Enfado & expresión del enfado a través de la agresión (Deater-Deckard et al., 2010; \\
& Deffenbacher, Lynch, Oetting, \& Swaim, 2002; Deffenbacher, Oetting, Lynch \& \\
& Morris, 1996; Gidron, Gaygisiz, \& Lajunen, 2014; Park, Enrigh, Essex, Zahn- \\
& Waxler, \& Klatt, 2013; Smith, Waterman, \& Ward, 2006; Stephens, Trawley, \& \\
& Ohtsuka, 2016; Walitzer, Deffenbacher, \& Shyhalla, 2015; Widger, 2012; Wyckoff, \\
& 2016) \\
& Rasgos de personalidad y comportamientos aberrantes al conducir (Beanland, \\
& Sellbom, \& Johnson, 2014) \\
& Specificidad situacional de rasgos de personalidad y su influencia en el \\
& comportamiento en la movilidad (Stephens \& Groeger, 2009) \\
& Agresión en la Movilidad la movilidad (Berdoulat, Vavassori, \& Sastre, 2013; \\
& Bogdan, Măirean, \& Havârneanu, 2016; Chliaoutakis et al., 2002; Deffenbacher et \\
& al., 2002; Dula \& Geller, 2003; Gidron et al., 2014; Gras et al., 2015; Kovácsová, \\
Movilidad & Stephens \& Sullman, 2014). \\
& \\
&
\end{tabular}


Tabla 2

Inventarios de Agresividad de Uso más Frecuente

\begin{tabular}{|c|c|}
\hline Inventario & Publicaciones \\
\hline Behavior Questionnaire & $\begin{array}{l}\text { (Amado et al., 2014; Beanland et al., 2014; Beneret al., } \\
\text { 2008; Berdoulat et al., 2013; Blankenship \& Nesbit, 2013; } \\
\text { Burdett, Charlton, \& Starkey, 2016; Newnam \& Von } \\
\text { Schuckmann, 2012; Qu, Ge, Jiang, Du, \& Zhang, 2014; } \\
\text { Rike, Johansen, Ulleberg, Lundqvist, \& Schanke, 2015; } \\
\text { Rosenbloom \& Wultz, 2011; Shahar, 2009; Sullman \& } \\
\text { Taylor, 2010; Warner \& Åberg, 2014; Zhang, Chan, \& } \\
\text { Zhang, 2015) }\end{array}$ \\
\hline Driving Anger Scale & $\begin{array}{l}\text { (Berdoulat et al., 2013; Björklund, 2008; Deffenbacher, } \\
\text { Petrilli, Lynch, Oetting, \& Swaim, 2003; Lajunen \& } \\
\text { Parker, 2001; Li et al., 2014; Przepiorka et al., 2014; Smith } \\
\text { et al., 2006; Stephens et al., 2016; Sullman \& Stephens, } \\
\text { 2013; Sullman, Stephens, \& Yong, 2014; Villieux } \\
\text { \&Delhomme, 2010; Zhang et al., 2015). }\end{array}$ \\
\hline $\begin{array}{l}\text { Driving Anger Expression } \\
\text { Inventory }\end{array}$ & $\begin{array}{l}\text { (Deffenbacher et al., 2002; Ge et al., 2015; Gras et al., } \\
\text { 2015; Herrero-Fernández, 2011a, 2011b, Sârbescu, 2012; } \\
\text { Sârbescu, Stanojević, \& Jovanović, 2014; Stephens \& } \\
\text { Sullman, 2014), }\end{array}$ \\
\hline Driving Behavior Survey & $\begin{array}{l}\text { (Clapp, Baker, Litwack, Sloan, \& Beck, 2014) (Baker, } \\
\text { Litwack, Clapp, Gayle Beck, \& Sloan, 2014; Przepiorka et } \\
\text { al., 2014), }\end{array}$ \\
\hline
\end{tabular}


Tabla 3

Frecuencias de Respuestas Obtenidas para el Primer Estudio.

Otras agresiones verbales

Insultos y/o etiquetas

Claxon o bocina

Bloquear el paso de otro vehículo

Amenazar con utilizar el vehículo para causar daño hacia otros vehículos o personas

Lenguaje no verbal de naturaleza agresiva (gestos o señas, tales como silbar, mirar, o escupir)

Comportamiento irrespetuoso y/o discriminatorio

Gritar

Invadir el flujo del tránsito agresivamente

Uso agresivo, excesivo o inadecuado de las luces altas

Meterse en la fila o conducir en sentido contrario para ahorrar tiempo

No respetar las leyes de tránsito y/o los señalamientos de tránsito (incluyendo semáforos)

Usar el vehículo para hacer contacto con otro vehículo o persona a manera de agresión (incluyendo daño físico)

No usar las direccionales antes de realizar una vuelta

Frenar a manera de agresión hacia el vehículo que viene detrás

Conducir a exceso de velocidad

No respetar el espacio entre los vehículos a manera de agresión

No ceder el paso en los cruces

Arrojar objetos

Rebasar por la izquierda

Contaminación auditiva

Impaciencia 
No respetar los espacios designados para personas discapacitadas 3

Mala educación $\quad 2$

No respetar a las autoridades 1

Responder agresivamente a las criticas $\quad 1$ 
Tabla 4

Adecuación Teórica de los Ítems Generados en el Primer Estudio

\section{Poca Consideración}

Bloquear el paso de otro vehículo

Invadir el flujo del tránsito agresivamente

Obstruir el tránsito peatonal

No respetar los espacios designados para el tránsito peatonal

No ceder el paso en los cruces

Meterse en la fila o conducir en sentido contrario para ahorrar tiempo

\section{Manifestación de Frustración/Enfado}

\section{Claxón o bocina}

Comportamiento irrespetuoso y/o discriminatorio

Gritar

Usar el vehículo para hacer contacto con otro vehículo o persona a manera de agresión (incluyendo daño físico)

Amenazar con utilizar el vehículo para causar daño hacia otros vehículos o personas Uso agresivo, excesivo o inadecuado de las luces altas

Agresión física o la amenaza de dicha agresión

Lenguaje no verbal de naturaleza agresiva (gestos o señas, tales como silbar, mirar, o escupir) Insultos y/o etiquetas

No respetar el espacio entre los vehículos a manera de agresión

Frenar a manera de agresión hacia el vehículo que viene detrás

Otras agresiones verbales

\section{Conducción de Riesgo}

No respetar las leyes de tránsito y/o los señalamientos de tránsito (incluyendo semáforos)

No usar las direccionales antes de realizar una vuelta

Conducir a exceso de velocidad 
Tabla 5

Índices Descriptivos de los Ítems del Segundo Estudio

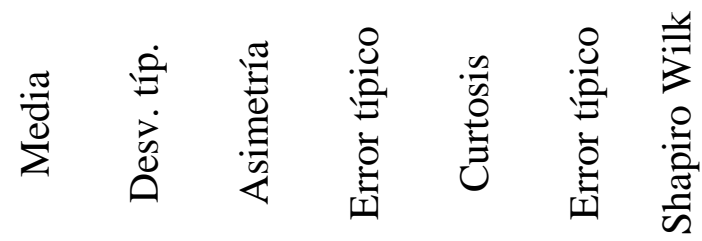

$\begin{array}{lllllllll}\text { No usar las direccionales antes de dar vuelta. } & 2.30 & 1.324 & .819 & .117 & -.473 & .234 & .829 * * *\end{array}$ $\begin{array}{llllllll}\text { Frenar para agredir al automóvil que viene } & 1.54 & .958 & 2.012 & .117 & 2.012 & .234 & .62 * * *\end{array}$ detrás.

Impedir el paso o no ceder el paso a otros vehículos.

No ceder el paso a un automóvil en las intersecciones ( 1 x 1$)$.

Proferir insultos o adjetivos calificativos a otras personas o automóviles.

No respetar los espacios para el tránsito peatonal.

Gritar a otras personas o automóviles. Agredir físicamente o amenazar con agredir a otras personas o automóviles.

Agredir con el vehículo a otras personas o automóviles.

Incorporarse por la fuerza al flujo de la circulación.

Utilizar el claxon o la bocina para indicar disgusto a otras personas o automóviles. Usar agresiva, excesiva o inadecuadamente las luces altas/largas. $\begin{array}{lllllll}2.11 & 1.039 & .921 & .117 & .407 & .234 & .842 * * *\end{array}$ $\begin{array}{lllllll}1.54 & .797 & 1.668 & .117 & .307 & .234 & .691 * * *\end{array}$

$\begin{array}{lllllll}1.82 & .954 & 1.127 & .117 & .858 & .234 & .79 * * *\end{array}$ $\begin{array}{lllllll}1.97 & 1.063 & 1.04 & .117 & .509 & .234 & .812 * * *\end{array}$ $\begin{array}{lllllll}1.81 & 1.008 & 1.176 & .117 & .808 & .234 & .771 * * *\end{array}$ $\begin{array}{lllllll}1.74 & 1.093 & 1.615 & 117 & 2.025 & .234 & .7 * * *\end{array}$ $\begin{array}{lllllll}1.75 & 963 & 1.209 & .117 & .844 & .234 & .761 * * *\end{array}$ $\begin{array}{lllllll}1.31 & .721 & 2.678 & .117 & 7.45 & .234 & .497 * * *\end{array}$ $\begin{array}{lllllll}1.32 & 689 & 2.586 & .117 & 7.459 & .234 & .521 * * *\end{array}$ $\begin{array}{lllllll}2.05 & .967 & .594 & .117 & -.371 & .234 & .852 * * *\end{array}$ 
$\begin{array}{lllllll}\text { No respetar el espacio entre los automóviles } & 1.5 & .846 & 1.956 .117 & 3.987 & .234 & .639 * * *\end{array}$ a manera de agresión.

$\begin{array}{llllllll}\text { Agredir de manera no verbal a otras personas } & 1.7 & .952 & 1.456 & .117 & 1.909 & .234 & .731 * * *\end{array}$ o automóviles (incluyendo gestos o señas, silbar, mirar agresivamente o escupir).

Agredir verbalmente a otras personas o automóviles.

Faltar al respeto o articular comentarios discriminatorios hacia niños, ancianos o mujeres.

$\begin{array}{lllllllll}\text { No respetar el reglamento de tránsito y/o los } & 1.79 & .92 & 1.635 & .117 & 2.091 & .234 & .769 * * *\end{array}$ señalamientos (incluyendo semáforos en rojo).

No ceder o impedir el paso a los peatones. $\begin{array}{llllll}1.54 & .874 & 1.96 & .117 & 4.081 & .234\end{array}$ $.656 * * *$ No respetar el orden de la fila y/o circular en sentido contrario para llegar más rápido.

Conducir a exceso de velocidad. $\begin{array}{lllllll}2.02 & .953 & .734 & .117 & .012 & .234 & .846^{* * *}\end{array}$ Hacer contacto, con el automóvil, a otro $\begin{array}{lllllll}1.25 & .683 & 3.433 & .117 & 13.23 & .234 & .422 * * *\end{array}$ automóvil o persona a manera de agresión (incluyendo daños físicos).

$\begin{array}{llllllll}1.69 & .954 & 1.434 & .117 & 1.638 & .234 & .73 * * *\end{array}$

$\begin{array}{lllllll}1.27 & 678 & 2.996 & .117 & 9.667 & .234 & .456^{* * *}\end{array}$ $\begin{array}{lllllll}1.47 & .815 & 2.119 & .117 & 4.877 & .234 & .622 * * *\end{array}$ Notas: $* * * p<.001$ 
Tabla 6

Análisis de Componentes Principales con Rotación Varimax para los Datos Obtenidos en el Segundo Estudio.

\begin{tabular}{|c|c|c|c|c|}
\hline & $\begin{array}{l}\text { Manifestaci } \\
\text { ón de } \\
\text { frustración/ } \\
\text { enfado }\end{array}$ & $\begin{array}{c}\text { Poca } \\
\text { Consideración }\end{array}$ & $\begin{array}{l}\text { Conducción } \\
\text { de riesgo }\end{array}$ & Comunalidades \\
\hline $\begin{array}{l}\text { Agredir con el vehículo a otras personas } \\
\text { o automóviles. }\end{array}$ & .822 & .119 & .052 & .691 \\
\hline $\begin{array}{l}\text { Agredir físicamente o amenazar con } \\
\text { agredir a otras personas o automóviles. }\end{array}$ & .818 & .096 & .039 & .657 \\
\hline $\begin{array}{l}\text { Hacer contacto, con el automóvil, a otro } \\
\text { automóvil o persona a manera de } \\
\text { agresión }\end{array}$ & .731 & .052 & 298 & .599 \\
\hline $\begin{array}{l}\text { Faltar al respeto o articular comentarios } \\
\text { discriminatorios hacia niños, ancianos o } \\
\text { mujeres. }\end{array}$ & .623 & .223 & .046 & .422 \\
\hline $\begin{array}{l}\text { No respetar el espacio entre los } \\
\text { automóviles a manera de agresión. }\end{array}$ & .542 & .363 & .227 & .449 \\
\hline $\begin{array}{l}\text { No ceder el paso a un automóvil en las } \\
\text { intersecciones }(1 \times 1) \text {. }\end{array}$ & .061 & .823 & .090 & .625 \\
\hline $\begin{array}{l}\text { Impedir el paso o no ceder el paso a } \\
\text { otros vehículos. }\end{array}$ & .156 & .751 & -.03 & .503 \\
\hline $\begin{array}{l}\text { No respetar los espacios para el tránsito } \\
\text { peatonal. }\end{array}$ & .237 & .645 & .233 & .527 \\
\hline $\begin{array}{l}\text { No respetar el reglamento de tránsito y/o } \\
\text { los señalamientos. }\end{array}$ & -.019 & .226 & .852 & .647 \\
\hline Conducir a exceso de velocidad. & .335 & -.014 & .685 & .465 \\
\hline
\end{tabular}


Tabla 7

Índices Descriptivos de los Ítems del Tercer Estudio

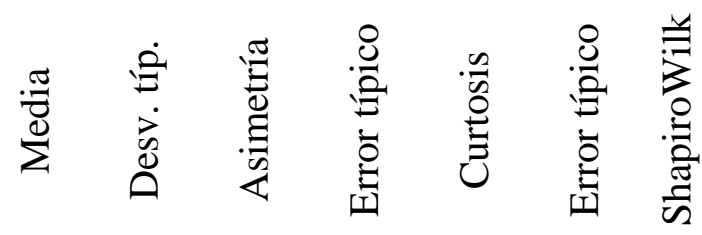

$\begin{array}{llllllll}\text { No usar las direccionales antes de dar vuelta. } & 2.34 & 1.385 & .768 & .058 & -.686 & .117 & .823 * * *\end{array}$ $\begin{array}{llllllll}\text { Frenar para agredir al automóvil que viene } & 1.56 & .937 & 1.877 & .058 & 3.203 & .117 & .64 * * *\end{array}$ detrás.

Impedir el paso o no ceder el paso a otros

$\begin{array}{lllllll}1.93 & 1.013 & 1.068 & .058 & .706 & .117 & .811 * * *\end{array}$ vehículos.

No ceder el paso a un automóvil en las

$\begin{array}{lllllll}2.03 & 1.111 & 1.007 & .058 & .335 & .117 & .819 * * *\end{array}$ intersecciones ( 1 x 1$)$.

$\begin{array}{lllllllll}\text { Proferir insultos o adjetivos calificativos a } & 1.88 & 1.038 & 1.087 & .058 & .548 & .117 & .792 * * *\end{array}$ otras personas o automóviles.

No respetar los espacios para el tránsito

$\begin{array}{lllllll}1.81 & 1.147 & 1.444 & .058 & 1.248 & .117 & .721 * * *\end{array}$ peatonal.

$\begin{array}{lllllllllll}\text { Gritar a otras personas o automóviles. } & & & 1.81 & .992 & 1.186 & .058 & .886 & .117 & .775^{* * *}\end{array}$ $\begin{array}{lllllllll}\text { Agredir físicamente o amenazar con agredir } & 1.45 & .865 & 2.14 & .058 & 4.231 & .117 & .58 * * *\end{array}$ a otras personas o automóviles.

Agredir con el vehículo a otras personas o $\begin{array}{lllllll}1.42 & .816 & 2.202 & .058 & 4.835 & .117 & .578 * * *\end{array}$ automóviles.

Incorporarse por la fuerza al flujo de la $\begin{array}{lllllll}2.04 & .999 & .799 & .058 & .134 & .117 & .845 * * *\end{array}$ circulación.

Utilizar el claxon o la bocina para indicar $\begin{array}{lllllll}2.13 & 1.07 & .879 & .058 & .216 & .117 & .846 * * *\end{array}$ disgusto a otras personas o automóviles. $\begin{array}{llllllll}\text { Usar agresiva, excesiva o inadecuadamente } & 1.67 & .851 & 1.565 & .058 & 2.159 & .117 & .714 * * *\end{array}$ las luces altas/largas. 
$\begin{array}{llllllll}\text { No respetar el espacio entre los automóviles } & 1.59 & .939 & 1.744 & .058 & 2.718 & .117 & .672^{* * * *}\end{array}$ a manera de agresión.

$\begin{array}{llllllll}\text { Agredir de manera no verbal a otras personas } & 1.7 & .958 & 1.439 & .058 & 1.704 & .117 & .734 * * *\end{array}$ o automóviles (incluyendo gestos o señas, silbar, mirar agresivamente o escupir).

Agredir verbalmente a otras personas o $\begin{array}{lllllll}1.7 & .976 & 1.471 & .058 & 1.72 & .117 & .727^{* * *}\end{array}$ automóviles.

Faltar al respeto o articular comentarios

discriminatorios hacia niños, ancianos o mujeres.

No respetar el reglamento de tránsito y/o los $\quad \begin{array}{lllllll}1.8 & 1.009 & 1.402 & .058 & 1.631 & .117 & .756^{* * *}\end{array}$ señalamientos (incluyendo semáforos en rojo).

No ceder o impedir el paso a los peatones. $\begin{array}{llllllll}1.65 & .947 & 1.716 & .058 & 2.36 & .117 & .685^{* * *}\end{array}$ $\begin{array}{llllllll}\text { No respetar el orden de la fila y/o circular en } & 1.61 & .957 & 1.757 & .058 & 2.765 & .117 & .676^{* * * *}\end{array}$ sentido contrario para llegar más rápido.

Conducir a exceso de velocidad.

Hacer contacto, con el automóvil, a otro automóvil o persona a manera de agresión (incluyendo daños físicos). $\begin{array}{lllllll}2.02 & 1.05 & .886 & .058 & .157 & .117 & .833^{* * * *}\end{array}$

$\begin{array}{lllllll}1.34 & .79 & 2.743 & 058 & 7.681 & .117 & .492^{* * * *}\end{array}$

Notas: $* * * p<.001$ 


\section{Tabla 8}

Análisis de Componentes Principales con Rotación Varimax para los Datos Obtenidos en el Tercer Estudio.

\begin{tabular}{|c|c|c|c|c|}
\hline & $\begin{array}{l}\text { Manifestació } \\
\mathrm{n} \text { de } \\
\text { frustración/e } \\
\text { nfado }\end{array}$ & $\begin{array}{c}\text { Poca } \\
\text { Consideración }\end{array}$ & $\begin{array}{l}\text { Conducción } \\
\text { de riesgo }\end{array}$ & Comunalidades \\
\hline $\begin{array}{l}\text { Agredir físicamente o amenazar con agredir } \\
\text { a otras personas o automóviles. }\end{array}$ & .819 & .220 & .138 & .723 \\
\hline $\begin{array}{l}\text { Agredir con el vehículo a otras personas o } \\
\text { automóviles. }\end{array}$ & .814 & .196 & .101 & .708 \\
\hline $\begin{array}{l}\text { Hacer contacto con el automóvil a otro } \\
\text { automóvil o persona a manera de agresión. }\end{array}$ & .777 & .138 & .199 & .683 \\
\hline $\begin{array}{l}\text { Faltar el respeto o articular comentarios } \\
\text { discriminatorios hacia niños, ancianos o } \\
\text { mujeres. }\end{array}$ & .765 & .15 & .172 & .638 \\
\hline $\begin{array}{l}\text { No respetar el espacio entre los automóviles } \\
\text { a manera de agresión. }\end{array}$ & .7 & .278 & .225 & .604 \\
\hline $\begin{array}{l}\text { Usar agresiva, excesiva o inadecuadamente } \\
\text { las luces altas/largas. }\end{array}$ & .658 & .215 & .298 & .55 \\
\hline Gritar a otras personas o automóviles. & .505 & .328 & .294 & .684 \\
\hline $\begin{array}{l}\text { No ceder el paso a un automóvil en las } \\
\text { intersecciones }(1 \times 1) \text {. }\end{array}$ & .135 & .844 & .123 & .611 \\
\hline $\begin{array}{l}\text { Impedir el paso o no ceder el paso a otros } \\
\text { vehículos. }\end{array}$ & .205 & .741 & .121 & .528 \\
\hline $\begin{array}{l}\text { No respetar los espacios para el tránsito } \\
\text { peatonal. }\end{array}$ & .325 & .629 & .153 & .54 \\
\hline Conducir a exceso de velocidad. & .246 & .078 & .839 & .592 \\
\hline $\begin{array}{l}\text { No respetar el reglamento de tránsito y/o los } \\
\text { señalamientos (incluyendo semáforos en } \\
\text { rojo). }\end{array}$ & .224 & .259 & .738 & .65 \\
\hline
\end{tabular}




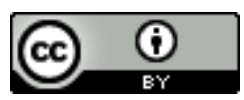

Este texto está protegido por una licencia CreativeCommons 4.0.

\begin{abstract}
Usted es libre para Compartir - copiar y redistribuir el material en cualquier medio o formato- y Adaptar el documento remezclar, transformar y crear a partir del material- para cualquier propósito, incluso comercialmente, siempre que cumpla la condición de:
\end{abstract}

Atribución: Usted debe reconocer el crédito de una obra de manera adecuada, proporcionar un enlace a la licencia, e indicar si se han realizado cambios. Puede hacerlo en cualquier forma razonable, pero no de forma tal que sugiera que tiene el apoyo del licenciante o lo recibe por el uso que hace. 